\title{
Rinitis y factores psicológicos: estado de su relación
}

\section{The association between rhinitis and psychological factors: an update}

\author{
Juan Carlos Fernández Rodríguez ${ }^{1}$, Mayte Herrera Mera ${ }^{2}$ \\ ${ }^{1}$ Bureau Veritas Centro Universitario, España. \\ ${ }^{2}$ Servicio de ORL, Hospital de Fuenlabrada, España.
}

Disponible online 30 de abril de 2013

\begin{abstract}
En el presente artículo se examina el concepto de rinitis, su clasificación y la posible justificación de los diversos tipos de rinitis como trastornos de tipo psicofisiológico. Además, se revisan las, hasta ahora, escasas investigaciones que han relacionado rinitis y factores psicológicos, investigaciones que en su mayoría tienen por objeto trastornos de tipo alérgico, entre las cuales podemos encontrar la rinitis. También se realiza una valoración del estado actual entre los factores psicológicos y la rinitis, se han estudiado principalmente la ansiedad, depresión y características de personalidad. Así mismo, se proponen futuras vías de estudio de la relación planteada.
\end{abstract}

Palabras clave: Rinitis; Variables Psicológicas; Ansiedad; Depresión.

The present article examines the concept of rhinitis, its classification and the reasons for classifying the different types of rhinitis as psychophysiological diseases. We review the few studies that have investigated the association between rhinitis and psychological factors. Most of these studies have focussed on allergies, among which is rhinitis. We also assess current knowledge on the association between rhinitis and psychological factors, mainly addressing anxiety, depression and personality characteristics. Further lines of research are suggested regarding this association.

Key words: Rhinitis; Psychological Variables; Anxiety; Depression.

Correspondencia: Juan Carlos Fernández Rodríguez, C/ Valportillo Primera nº 22. 28108 - Alcobendas.

E-mail: juan-carlos.fernandez@es.bureauveritas.com. E-mail de la co-autora: mayteherreramera@hotmail.com 
El término rinitis se aplica generalmente a la alteración inflamatoria de la mucosa de la fosa nasal (Mazón, Nieto y Uixera, 2011), a diferencia de la inflamación de la mucosa paranasal que determina que a menudo se trate de sinusitis (Penha, 1992). Las manifestaciones clínicas de la rinitis incluyen a menudo obstrucción nasal, secreción acuosa y sensación de plenitud nasal, cosquilleo, escozor (que en algunos casos llega a extenderse a toda la cabeza), estornudos, y de forma eventual, conjuntivitis. La rinitis no es una enfermedad que ponga en peligro la vida del paciente, pero a menudo origina molestias severas e incapacidad, reducción de la calidad de vida y trastornos de sueño (Camelo y Solé, 2010). Así, en niños con síntomas no controlados, puede ocasionar alteraciones del sueño (Young, Finn y Kim, 1997) y problemas de aprendizaje escolar (Walter, Khan-Wasti, Fletcher, Cullinan, Harris y Sheik 2007), en adultos, los síntomas nasales pueden alterar las actividades diarias, con un empeoramiento de la capacidad de concentración y dificultades en las relaciones sociales y en el sueño (Mora, 2004).

La etiología de la rinitis es múltiple, su origen puede deberse a una infección, a causas alérgicas, alteraciones vegetativas, cambios bruscos de temperatura, sustancias irritantes dispersas, estrés y otros agentes emocionales, etc. En numerosas ocasiones la rinitis puede deberse a una combinación entre varios de estos factores.

En la bibliografía se pueden encontrar numerosas taxonomías de la rinitis, clasificaciones que principalmente están realizadas en función de los factores desencadenantes o inductores del trastorno. Una clasificación muy útil es la propuesta por Becker, Nauman y Pfaltz (1992); según esta clasificación existen cuatro tipos de rinitis:

a) Rinitis aguda: es consecuencia de una infección vírica o eventualmente de una infección bacteriana secundaria. Es la llamada coriza, catarro común o resfriado común. Existen cerca de unos 200 virus causantes del resfriado común (Hilger, 1993).

b) Rinopatía alérgica: es la rinitis alérgica. Es el resultado de una respuesta de hipersensibilidad tipo I a diversos alérgenos, las reacciones de tipo I también se conocen como reacciones de hipersensibilidad inmediata, están mediadas por IgE o inmonoglobulina E, tipo de anticuerpo que media en las reacciones alérgicas (Sánchez-Guerrero, 2004). Dentro de las manifestaciones alérgicas posibles encontramos la fiebre del heno o polinosis, la forma más común de todas. Este tipo de rinitis se divide a su vez en rinopatía alérgica estacional (producida por el polen) y en rinopatía alérgica perenne o aperiódica, en este segundo tipo de rinitis se debe a la presencia de alérgenos inhalatorios (polvo, pelos de animal, hongos, etc.), alimentarios (pescado, mostaza, leche, etc.), alérgenos bacterianos, parásitos o de otro tipo, además, esta clase de rinitis es independiente de la época del año.

c) Rinopatía vasomotora, rinitis vasomotora o rinitis idiopática: esta rinitis es similar a la rinopatía alérgica aperió- dica, casi siempre presenta un curso paroxístico de forma clara. Esta forma de rinitis se debe a alteraciones vegetativas (sobre todo de carácter parasimpático) de los vasos que inervan la mucosa nasal. En este tipo de rinitis no se ha podido demostrar la presencia de alérgenos o de anticuerpos específicos (Tran, Vickery y Blaiss, 2011).

d) Formas crónicas: son otras rinitis de carácter crónico, como pueden ser la rinitis seca anterior, la rinopatía gravídica o la rinitis atrófica y ocena.

En cuanto a datos epidemiológicos existe gran confusión en las cifras, por lo que no existen datos demasiado detallados, tampoco existen datos exactos para la población española. En muchas ocasiones, la persona que sufre la enfermedad no acude a la consulta del médico, siendo éste un factor que hace difícil calcular con exactitud datos epidemiológicos en este trastorno. En Estados Unidos la rinitis alérgica afecta del 10 al 30\% de la población adulta y al $30 \%$ de población infantil (Tran, Vickery y Blaiss, 2011), lo cual puede llegar a suponer al menos unos 40 millones de pacientes en Estados Unidos cada año. En España, en un estudio con niños de entre 4 a 17 años, se estimó una prevalencia de la rinitis alérgica de $6.8 \%$ de la población general (Muñoz, 2005).

Aunque la posible conexión entre los distintos tipos de rinitis y factores psicológicos no constituye una relación bien establecida, puesto que apenas existe investigación básica, sí existen argumentos que apoyan una relación positiva. En el caso de las enfermedades alérgicas o de tipo inmunitario se ha señalado frecuentemente la presencia de factores psicológicos (Camelo, 2005), en nuestros días existen datos suficientes para afirmar que bajo estados emocionales negativos es más probable desarrollar enfermedades relacionadas con el sistema inmune o adquirir hábitos poco saludables que hagan minar la salud (Cano-Vindel, Sirgo y Diaz-Ovejero, 1999), incluso existe evidencia entre las exacerbaciones alérgicas en personas predispuestas y estrés, llegando los factores estresantes a inducir cambios emocionales y de afrontamiento, cambios que pueden llegar a modificar la función inmunológica (Belloch, Sandín y Ramos, 2009). En este sentido, son ya clásicos los trabajos pioneros en el área de la psicoinmunología (Ader, 1981; Ader y Cohen, 1985, 1993; Solomon, 1987) que relatan la relación entre sistema inmune y factores psicológicos. Se ha apuntado a cómo la ansiedad puede estar relacionada con los síntomas de alergia (Retamales, 1998; Rosenbaum y Pollack, 1987;) y cómo los pacientes con trastornos alérgicos (rinitis entre ellos) pueden sufrir ansiedad o depresión a causa de su enfermedad alérgica (Buela-Casal, Santos, Carretero y Cachinero, 2002, Michel, 1994), además, las enfermedades de tipo alérgico pueden guardar relación con la agorafobia y los trastornos de pánico, en particular en los pacientes que han sufrido anafilaxia (Schmidt-Traub,1995). Incluso en el caso de las rinitis agudas ha sido descrita la influencia de las emociones negativas en la probabilidad de aparición de enfermedades infecciosas (Cohen y Williamson, 1991). 
En el caso de la rinitis vasomotora y como acción predominante del parasimpático, este trastorno puede no ser ajeno al influjo de las variables psicológicas como se ha demostrado en otros trastornos de carácter predominantemente parasimpático como el asma bronquial (Fernández, 2003, Isemberg, Lehrer y Hochron, 1992).

Por tanto, la rinitis se podría considerar como un trastorno de tipo psicofisiológico, no siendo este trastorno una excepción al resto de los trastornos de esta clase, en los cuales podemos encontrar factores psicológicos íntimamente relacionados con el mantenimiento del trastorno. Los factores psicológicos (sobre todo emocionales) aparecen citados en la rinitis como estímulos desencadenantes, incluso en la literatura de carácter biomédico, ya sea en el ámbito de rinopatía alérgica (Gavilán, 1989) o de rinopatía vasomotora (Pulido, 1992).

\section{Método}

Se ha realizado una búsqueda de bibliografía en varias bases de datos, en concreto se han consultado: Psicodoc, Cindoc, Dialnet, Psycarticles y Google académico. Los descriptores utilizados han sido: rinitis, ansiedad, depresión y variables psicológicas, además de sus equivalentes en inglés. La búsqueda bibliográfica se realizó en junio de 2012, no estableciéndose restricciones en cuanto al periodo de tiempo consultado.

\section{Resultados}

La investigación de carácter psicológico existente en torno a la rinitis no es demasiado extensa y en muchas ocasiones poco clara, hecho que se traduce en muestras muy escasas y en una mezcla de varios tipos de pacientes en las diversas investigaciones (ya que es muy frecuente la realización de estudios con pacientes de tipo alérgico), riníticos, asmáticos, pacientes con urticaria, etc. Estos hechos hacen que se tengan que tomar con cautela las conclusiones de la mayoría de los estudios.

La investigación básica en cuanto a procesos psicológicos también es muy escasa, existiendo algún estudio que comprueba positivamente la posibilidad de la aparición de crisis riníticas a través del condicionamiento clásico (Gauci, Husband, Saxarra y King, 1994). No se han encontrado estudios referentes a otros procesos psicológicos como por ejemplo el condicionamiento operante. En cuanto a factores de tipo emocional, sólo la ansiedad y en menor medida la depresión, han sido objeto de estudio en algunos casos. En cuanto a la ansiedad, es difícil encontrar estudios en la literatura que investiguen la relación de ésta emoción exclusivamente en la rinitis, ya que la mayoría de las investigaciones, los estudios se realizan junto con otros trastornos de origen alérgico. Unal, Berksun, Kinikli y Kaya (1991) encontraron en una muestra de 23 pacientes alérgicos y 25 sujetos sanos y a través de autoinformes, una mayor prevalencia de síntomas de ansiedad en los sujetos alérgicos, aunque la escasa muestra empleada y la mezcla de pacientes con rinitis y urticaria en el grupo experimental hacen que los datos no sean definitivos. Hart, Lahey, Hynd y Loeber (1995) examinaron los datos de un estudio longitudinal de tres años de duración, realizado con 177 pacientes externos de un hospital, todos ellos sujetos en edad infantil. En este estudio se entrevistó, de forma anual, a los propios sujetos, a los padres y a los profesores de los niños. Las conclusiones sugieren que la rinitis alérgica guarda relación con la ansiedad y que la emoción es mayor en aquellos sujetos que sufren desde hace más tiempo la enfermedad. Addolorato, Ancona, Capristo, Grazziosetto, Di Rienzo, Maurizi y Gasbarrini (1999) obtuvieron diferencias estadísticamente significativas al evaluar ansiedad entre 45 mujeres con rinitis ( 24 con rinitis alérgica y 21 con rinitis vasomotora) y 64 mujeres sanas no alérgicas de un grupo control, emparejadas en edad y características sociodemográficas. La emoción se midió a través del STAI de Spielberger, las medidas de ansiedad fueron significativamente mayores en el grupo de rinitis tanto en rasgo como en estado de ansiedad. Buela-Casal et al. (2002), realizó un estudio cuasi-experimental transversal, con un grupo de 22 sujetos alérgicos al polen y 206 sujetos no alérgicos, todos ellos militares internados. Se encontraron diferencias entre ambos grupos en ansiedad de tipo social medida a través de la Escala de Evitación y Angustia Social (EEAS) y de la Escala de Temor a la Evaluación Negativa (ETEN), y diferencias en ansiedad general a través del STAI (diferencias en estado pero no en rasgo, aunque las puntuaciones tienden a la significación). Kiecolt-Glaser et al. (2009), en una muestra compuesta por 28 pacientes con rinitis alérgica y ante dos situaciones, una de ellas estresante y la otra sin dicho matiz, encontraron como la ansiedad y el estrés pueden modular las pruebas diagnósticas de punción cutánea, proporcionando un mecanismo mediante el cual la tensión puede favorecer la producción de IgE y como la ansiedad puede aumentar y prolongar la sintomatología de la enfermedad. Sansone y Sansone (2011), en una revisión de artículos, a partir de las bases de datos PubMed y PSycINFO, hallaron que en 9 de los 11 artículos encontrados, y a pesar de deficiencias metodológicas, indican una asociación entre rinitis alérgica y síndrome de ansiedad, además, parece que hay un número de variables posibles que intervienen en la relación entre las alergias y la ansiedad, por ejemplo, las alergias pueden aumentar el riesgo de estos síndromes mediante la activación del sistema inmune, o la afectación del sueño en los alérgicos a través de la obstrucción nasal, incluso las alergias pueden afectar negativamente el funcionamiento cognitivo y contribuir a la perturbación psiquiátrica. Lv, Xi, Han y Zang (2010) investigaron el estado psicológico de 337 adultos con rinitis alérgica en temporada de alergia, mediante autoinforme (SCL-90), encontraron que las puntuaciones de los pacientes con rinitis son estadísticamente más altas que las de los sujetos no alérgicos en términos de ansiedad, no encontraron diferencias significativas en cuanto a sexo y edad. Runeson, Wahlstedt y Norback (2011), en un estudio sobre 193 personas, que trabajaban en 19 edificios diferentes y con posibles problemas con el aire acondicionado, y a través de pruebas de personalidad (Karolinska Scales of Personality, KSP) y cuestionarios posta- 
les, informaron que la rinitis no atópica se asoció con puntuaciones en varias escalas relacionados con la ansiedad, mientras que la rinitis atópica no se asoció con puntuaciones en dichas escalas.

El estudio de otras emociones y factores psicológicos ha sido más bien escaso. En cuanto a la depresión, es de señalar el estudio de Bell, Jasnoski, Kagan y King (1991), en esta investigación se utilizó una muestra no clínica de 379 estudiantes postgraduados y se comprobó que el $71 \%$ de los sujetos que habían recibido el diagnóstico profesional de depresión tenían una amplia historia de enfermedad alérgica, así mismo, los sujetos no alérgicos no difieren en la percepción de la frecuencia de la depresión. Addolorato et al. (1999) en el mismo grupo descrito anteriormente y a través de la escala para la depresión de Zung no encontraron puntuaciones significativamente mayores en el grupo de mujeres con rinitis en comparación con el grupo de mujeres control. Marshall, O’Hara y Steinberg (2002), en un estudio de cuatro años en los que participaron un total de 98 pacientes con rinitis y 89 sujetos control, equilibrados en cuanto a edad, inteligencia, sexo y años de educación, se midió el estado de ánimo mediante la Positive Affect Negative Affect Scales (PANAS), también se midió la fatiga o cansancio (físico y mental) mediante el Multidimensional Fatigue Inventory (MFI-20); los pacientes alérgicos informaron de mayores niveles de fatiga general y mental, así como una reducción de la motivación, describiendo los pacientes sentimientos de tristeza y escaso bienestar, no se encontró reducción de la fatiga física. Sansone y Sansone (2011), en su estudio de revisión, ya citado anteriormente, encontraron que en 10 de los 12 estudios encontrados sobre síndromes alérgicos se establecía una relación entre alergia y estado de ánimo, aunque muchos de los estudios poseen importantes deficiencias metodológicas.

En cuanto al estudio de las características de personalidad en sujetos que sufren rinitis, Bell, Jasnoski, Kagan y King (1990) estudiaron la relación de este trastorno y otras enfermedades alérgicas con la timidez extrema en un amplio grupo compuesto de 375 estudiantes postgraduados, encontraron que los sujetos más introvertidos presentaban puntuaciones significativamente más altas en los autoinformes de depresión, miedo y fatiga, junto con puntuaciones más elevadas en rinitis alérgica, pero no en otros trastornos alérgicos. Gauci, King, Saxarra, Tullocch y Husband (1993) con un grupo de 22 mujeres con rinitis alérgica y 18 no alérgicas, con edades entre los 19 y los 68 años encontraron, utilizando el M.M.P.I., que las mujeres alérgicas puntuaban significativamente más alto en las escalas de Hipocondría (Hs) e Introversión social ( $\mathrm{Si}$ y significativamente más bajas en el Factor corrector (K) y en la escala adicional Fuerza del yo (Es), resultados que pueden mostrar que las mujeres con rinitis alérgica perenne tengan un funcionamiento psicológico más pobre que las no alérgicas. McGee, Stanton y Sears (1993) estudiaron desórdenes alérgicos y déficit de atención e hiperactividad en niños alérgicos, para ello, utilizaron una muestra de 1037 niños entre 9 y 13 años (con rinitis, asma, eczema y urticaria) y evaluaron mediante autoinforme conductas de hiperactividad en padres y profesores, encontrando que los trastornos no guardan relación entre sí. También encontraron en los niños de 13 años que los niveles de inmonuglobulina $\mathrm{E}$ ( $\mathrm{IgE}$ ) y la prueba cutánea de respuesta atópica o Prick-Test no guardan relación con los informes de hiperactividad e inatención. En el estudio descrito con anterioridad, Buela-Casal et al. (2002) midieron la personalidad con el Cuestionario de Personalidad de Eysenck (EPQ-A), obtuvieron diferencias estadísticamente significativas en las subescalas de extroversión y neuroticismo, las demás escalas no se diferencian significativamente entre los grupos. Muluk, Oguzturk, Koç y Ekici (2003) en un estudio con 29 pacientes con rinitis alérgica y 29 sujetos control, utilizando el M.M.P.I encontraron puntuaciones significativamente más altas en las escalas de Depresión (D), Paranoia (Pa), Introversión Social (Si), Hipocondría (Hs) e Histeria (Hy), siendo las puntuaciones más altas en mujeres que en hombres. Ryden, Andersson y Andersson (2007) evaluaron los rasgos de personalidad de 63 pacientes con rinitis mediante las Karolinska Scales of Personality (KSP) y se compararon con un grupo de 400 sujetos equilibrados en cuanto a edad y sexo, el grupo de pacientes difieren en una serie de factores como desinhibición, una tendencia a no expresar emociones, dificultad para el mantenimiento de una distancia emocional con los demás y agresividad, un patrón que sugiere que se exponen a una estimulación mayor a la que pueden procesar en términos de pensamiento y sentimientos, lo cual genera gran excitación fisiológica que puede contribuir a sus reacciones de intolerancia. Lv, Han, Xi y Zhang (2010) investigaron las relaciones de la rinitis alérgica (moderada a severa) y rasgos de personalidad en 52 pacientes ambulatorios no psiquiátricos y de sexo femenino, los rasgos de personalidad fueron evaluados con el M.M.P.I., el grupo de alergia puntuó significativamente más alto en las escalas de Hipocondría (Hs), Depresión (D), Histeria (Hy), Psicastenia $(\mathrm{Pt})$, Esquizofrenia $(\mathrm{Sc})$, Introversión Social $(\mathrm{Si})$ y una escala de validez (F), correlacionando positivamente además con pruebas cutáneas.

\section{Discusión}

A la vista de los estudios examinados, parecen existir datos que apuntan en la dirección que la rinitis puede estar relacionada con variables de carácter psicológico. Las investigaciones existentes en nuestros días, aunque no presentan afirmaciones concluyentes, sí parece que apuntan en ese sentido, sobre todo en el caso del estudio de la ansiedad, conclusión que se apunta en el estudio de Hart et al. (1995), una de las investigaciones más rigurosas de las revisadas.

Existen estudios empíricos que apuntan hacia la presencia de relación entre factores psicológicos, sobre todo emocionales, y enfermedad alérgica (Klinger, Herrera, Díaz, Jhann, Ávila y Tobar, 2005). El mayor problema con que nos encontramos es la presencia de diversos tipos de trastornos (asma, rinitis, urticaria, etc.) en una misma muestra, seguido en muchos casos de 
muestras clínicas muy escasas en cuanto a número de participantes.

Las emociones negativas parece que pueden promover respuestas alérgicas inflamatorias (Katon, Richardson, Lozano y McCauley, 2004). Aunque los datos apuntan en un sentido positivo, es difícil en estos estudios determinar con exactitud la posible influencia de variables psicológicas en la rinitis exclusivamente, pareciendo más que probable establecer esa relación cuando evaluamos a pacientes con trastorno alérgico en general (Retamales, 1998).

También sería conveniente realizar estudios en rinitis de tipo vasomotor, variante de la rinitis de la que apenas existen estudios, sobre todo para comprobar su diferencia o no de la rinitis alérgica, en este sentido Addolorato et al. (1999) compararon entre sí las dos variantes del trastorno (alérgica y vasomotora) de su grupo experimental y no encontraron diferencias en rasgo de ansiedad, estado de ansiedad y depresión.

En resumen, parece que la rinitis puede guardar relación con variables de índole psicológica, al modo de los trastornos psicofisiológicos, pero para afirmar de modo satisfactorio y firme esta relación se necesitan investigaciones más concretas, empezando por estudios descriptivos, con escalas más exactas y fiables, y muestras más amplias, para más tarde realizar investigaciones que estudien la capacidad que tienen los factores psicológicos, conductuales y emocionales de afectar al grado y la frecuencia de inflamación de la mucosa nasal y por tanto de poder modificar los índices de morbilidad del trastorno.

\section{Referencias}

1. Ader, R. (1981). Psychoneuroinmunology. New York: Academic Press.

2. Ader, R. y Cohen, N. (1985). CSN-inmune system interactions: Conditioning phenomena. Behavioral and Brain Sciences, 8, 379-426. http://dx.doi.org/10.1017/ $\underline{\mathrm{S} 0140525 \mathrm{X} 00000765}$

3. Ader, R. y Cohen, N. (1993). Psychoneuroinmunology. Conditioning and stress. Annual Review of Psychology, 44, 55-85. http://dx.doi.org/10.1146/annurev. ps.44.020193.000413

4. Addolorato, G., Ancona, C., Capristo, E., Graziosetto,R., Di Rienzo, L., Maurizi, M. y Gasbarrini, G. (1999). State and trait anxiety in women affected by allergic and vasomotor rhinitis. Journal of Psychosomatic Research, 46, 283289. http://dx.doi.org/10.1016/S0022-3999(98)00109-3

5. Becker, W., Nauman, H.H. y Pfaltz, C.R. (1992). Otorrinolaringología. Barcelona: Mosby-Doyma.

6. Bell, I.R., Jasnosky, M.L., Kagan, J. y King, D. (1990). Is allergic rhinitis more frequent in young adults with extreme shyness? A preliminary survey. Psychosomatic Medicine, 52, 517-525.

7. Bell, I.R., Jasnoski, M.L., Kagan, J. y King, D. (1991). Depression and allergies: Survey of a nonclinical population. Psychoteraphy and Psychosomatic, 55, 24-31. http://dx.doi.org/10.1159/000288404

8. Belloch, A., Sandin, B. y Ramos, F. (2009). Manual de Psicopatología. Madrid: McGraw Hill.

9. Buela-Casal, G., de Los Santos, M., Carretero, H. y Cachinero, J. (2002). Análisis de la interrelación entre alergia y variables psicológicas. Salud Mental, 25, 23-28.

10. Camelo, S. (2005). Psiconeuroinmunología, breve panoramica. Diversitas, $1,148-160$.

11. Camelo, I.C y Solé, D (2010). Allergic rhinitis. Indicators of quality of life. Journal Brasileiro de Pneumologia, 36, 124-133.

12. Cano-Vindel, A., Sirgo, A. y Diaz-Ovejero, M.B. (1999). Control, defensa y expresión de emociones: Relaciones con salud y enfermedad. En E.G. Fernández-Abascal y F. Palmero (Coord.), Emociones y Salud (pp. 69-87). Barcelona: Ariel.

13. Cohen, N. y Williamson, G.M. (1991). Stress and infectious disease in humans. Psychological Bulletin, 109, 5-24. http://dx.doi.org/10.1037/0033-2909.109.1.5

14. Fernández, J.C. (2003). Emociones y asma bronquial. Madrid: Servicio de Publicaciones de la Universidad Complutense de Madrid.

15. Gavilan, C. (1989). Pregrado. Otorrinolaringología, Tomo I. Madrid: Luzán 5.

16. Gauci, M., Husband, M.G., Saxarra, H. y King, M.G. (1994). Paulovian conditioning of nasal tryptase in human subjects with allergic rhinitis. Physiology and Behavior, 55, 823-825. http://dx.doi.org/10.1016/0031-9384(94)90066-3

17. Gauci, M., King, M.G., Saxarra, H., Tullocch, B.J. y Husband, A.J. (1993). A Minnesota Multiphasic Personality Inventory profile of women with allergic rhinitis. Psychosomatic Medicine, 55, 533-540.

18. Hart, E.L., Lahey, B.B., Hynd, G.W. y Loeber, R. (1995). Association of chronic overanxious disorder with atopic rhinitis in boys: A four year longitudinal study. Journal of Clinical Child Psychology, 24, 332-337. http://dx.doi. org/10.1207/s15374424jecp2403_10

19. Hilger, P.A. (1993). Enfermedades de la nariz. En G.L. Adams, L.R. Boies y P.A. Hilger (Eds.), Otorrinolaringología de Boies (pp. 218-249). México D.F.: InteramericanaMcGraw Hill.

20. Isemberg, P.M., Lehrer, P.M. y Hochron, S.M. (1992). The effects of suggestion and emotional arousal in pulmonary function in asthma: A review and hypothesis vagal mediation. Psychosomatic Medicine, 54, 192-216.

21. Katon, W.J, Richardson, L., Lozano, P. y McCauley, E. (2004). The relationship of asthma and anxiety. Psychosomatic Medicine, 66, 349-355. http://dx.doi.org/10.1097/01. psy.0000126202.89941.ea

22. Kielcolt-Glaser, J.K., Heffner, K.L., Glaser, R., Malarkey, W.B., Porter, K., Atkinson, C., Laskowski, S.L. y Marshall, G.D. (2009). How stress and anxiety can alter inmediate and late phase skin test response in allergic rhinitis. 
Psychoneuroendocrinology, 34, 670-680. http://dx.doi. org/10.1016/j.psyneuen.2008.11.010

23. Klinger, J.C., Herrera, J.A., Díaz, M.L., Jhann, A.A., Ávila, G.I. y Tobar, C.I. (2005). La psiconeuroinmunología en el proceso de salud enfermedad. Colombia Médica, 36, 120129.

24. Lv, X., Han, D., Xi, L. y Zhang, L. (2010). Psychological aspects of female patients with moderate to severe persistent allergic rhinitis. Journal for Otorhinolaryngology, Head and Neck Surgery, 72, 235-241.

25. Lv, X., Xi, L., Han, D. y Zhang, L. (2010). Evaluation of the psychological status in seasonal allergic rinitis patients. Journal for Otorhinolaryngology, Head and Neck Surgery, 72, 84-90.

26. Marshall, P.S., O’Hara, C. y Steinberg, P. (2002). Effects of seasonal allergic rinitis on fatigue levels and mood. Psychosomatic Medicine, 64, 684-691. http://dx.doi. org/10.1097/01.PSY.0000021944.35402.44

27. Mazon, A., Nieto, A. y Uixera, S. (2011). Actualización: Alergia de las vías respiratorias altas: rinitis, faringitis, tos crónica y otitis media. Anales de Pediatría Continuada, 9, 81-88.

28. McGee, R., Stanton, W.R. y Sears, M.R. (1993). Allergic disorders and attention deficit disorder in children. Journal of Abnormal Child Psychology, 21, 79-88. http://dx.doi. org/10.1007/BF00910490

29. Michel, F.B. (1994). Psychology of the allergic patient. Allergy, 49, 28-30. http://dx.doi. org/10.1111/j.1398-9995.1994.tb04235.x

30. Mora, A. (2004). Definición y clasificación de la rinitis. En J.M. Negro (Ed.), Rinitis Alérgica, mecanismos y tratamiento (pp.135-141). Mataró: MRA Ediciones.

31. Muñoz, F. (2005). Rinitis alérgica y patología asociada. Pediatría Integral, 7, 531-543.

32. Muluk, N.B., Oguzturk, O., Koç, C. y Ekici, A. (2003). Minnesota multiphasic personality inventory profile of patients with allergic rhinitis. Journal of Otolaryngology, 32, 198-202. http://dx.doi.org/10.2310/7070.2003.40380

33. Penha, R. (1992). Rinitis agudas y crónicas. En P. Abello y J. Traserra (Eds.), Otorrinolaringología (344-348). Barcelona: Doyma.

34. Pulido, M.V. (1992). Rinitis alérgicas y vasomotoras. En P. Abello y J. Traserra (Eds.), Otorrinolaringología (344348). Barcelona: Doyma.

35. Retamales, R. (1998). Factores psicológicos y rasgos de personalidad de enfermos alérgicos referidos a interconsulta. Psicología.COM, 2. Disponible en: http://www.psiquiatria.com/psicologia/vol2num1/art $4 . \mathrm{htm}$
36. Rosenbaum, M. y Pollack, M.H. (1987). Anxiety. En T.P. Hacket y N.H. Cassem (Eds.), Handbook of General Hospital Psychiatry (154-183). Massachusets: PSG, Littleton.

37. Runeson, R., Wahlstedt, K. y Norback, D. (2011). Pilot study of personality traits assessed by the Karolinska Scales of Personality (KSP) in asthma, atopy, and rhinitis. Perceptual and Motor Skills, 113, 909-920. http://dx.doi. org/10.2466/02.09.15.PMS.113.6.909-920

38. Ryden, O., Anderson M. y Anderson, B. (2007). Personality: A vulnerability factor in rinitis?. Psychology, Health and Medicine, 12, 328-333. http://dx.doi. org/10.1080/13548500600636873

39. Sánchez-Guerrero, I. (2004). Patogenia de la rinitis alérgica. En J.M. Negro (Ed.), Rinitis Alérgica, mecanismos y tratamiento (153-164). Mataró: MRA Ediciones.

40. Sansone, R.A y Sansone, L.A. (2011). Allergic Rhinitis. Relationships with Anxiety and Mood Syndromes. Innovations in Clinical Neuroscience, 8, 12-17.

41. Schmidt-Traub, S. (1995). Das psychoimmunologische netzwerk von panisktorung, agorapohobie und allesgischer reaktion. Therapeutische Umschau, 52, 123-128.

42. Solomon, G.F. (1987). Psychoneuroimmunology: Interactions between central nervous system and immune system. Journal of Neuroscience Research, 18, 1-9. http://dx.doi.org/10.1002/jnr.490180103

43. Tran, N.P., Vickery, J y Blaiss, M.S. (2011). Management of rhinitis: allergic and non-allergic. Allergy Asthma Inmunology, 3, 148-156. http://dx.doi.org/10.4168/ aair.2011.3.3.148

44. Unal, S., Berksun, O., Kinikli, G. y Kaya, E. (1991). Kronik urtiker ve allerjik nezle olgularinda psikolojik semptomlar. Turk Psikiyatri Dergisi, 2, 289-293.

45. Walker, S., Khan-Wasti S., Fletcher M., Cullinan P., Harris J. y Sheikh A. (2007). Seasonal allergic rhinitis is associated with a detrimental effect on examination performance in United Kingdom teenagers: case-control study. Journal of Allergy Clinical Immunology, 120, 381-387. http:// dx.doi.org/10.1016/j.jaci.2007.03.034

46. Young, T., Finn, L. y Kim, H. (1997). Nasal obstruction as a risk factor for sleep-disordered breathing. The University of Winsconsin Sleelp and Respiratory research Group. Journal of Allergy and Clinical Inmunology, 99, 757-762. http://dx.doi.org/10.1016/S0091-6749(97)70124-6

Fecha de recepción: 28 de marzo de 2012

Fecha de recepción de la primera versión modificada: 6 de de septiembre de 2012

Fecha de aceptación: 27 de septiembre de 2012 